University of New Hampshire

University of New Hampshire Scholars' Repository

Space Science Center

Institute for the Study of Earth, Oceans, and

Space (EOS)

2000

\title{
Estimation of GRB detection by FiberGLAST
}

\author{
Surasak Phengchamnan \\ University of Alabama - Huntsville \\ K Aisaka \\ University of California - Los Angeles \\ Muzaffer Atac \\ University of California - Los Angeles \\ W Robert Binns \\ Washington University in St Louis \\ J Buckley \\ Washington University in St Louis
}

See nextpage for additional authors Follow this and additional works at:https://scholars.unh.edu/ssc

Part of the Astrophysics and Astronomy Commons

\section{Recommended Citation \\ Estimation of GRB detection by FiberGLAST Phengchamnan, S. and Aisaka, K. and Atac, M. and Binns, W. R. and Buckley, J. H. and Cherry, M. L. and Cline, D. and Dowkontt, P. and Epstein, J. W. and Finger, M. H. and Fishman, G. J. and Guzik, T. G. and Hink, P. L. and Israel, M. H. and Kappadath, S. C. and Karr, G. R. and Kippen, R. M. and Macri, J. and Mallozzi, R. S. and McConnell, M. L. and Pischalnikov, Y. and Paciesas, W. S. and Parnell, T. A. and Pendleton, G. N. and Preece, R. D. and Richardson, G. A. and Rielage, K. and Ryan, J. M. and Stacy, J. G. and Tümer, T. O. and Wallace, D. B. and Wilson, R. B., AIP Conference Proceedings, 526, 706-710 (2000), DOI:http://dx.doi.org/10.1063/1.1361626}

This Conference Proceeding is brought to you for free and open access by the Institute for the Study of Earth, Oceans, and Space (EOS) at University of New Hampshire Scholars' Repository. It has been accepted for inclusion in Space Science Center by an authorized administrator of University of New Hampshire Scholars' Repository. For more information, please contact Scholarly.Communication@unh.edu. 


\section{Authors}

Surasak Phengchamnan, K Aisaka, Muzaffer Atac, W Robert Binns, J Buckley, M L. Cherry, David B. Cline, Paul F. Dowkontt, John W. Epstein, M H. Finger, Gerald J. Fishman, T G. Guzik, P L. Hink, Martin H. Israel, S C. Kappadath, Gerald Karr, R M. Kippen, John R. Macri, Robert S. Mallozzi, Mark L. McConnell, Yuriy Pischalnikov, W Paciesas, Thomas A. Parnell, Geoffrey N. Pendleton, R D. Preece, Georgia A. Richardson, Keith R. Rielage, James M. Ryan, J G. Stacy, O T. Tumer, Donald B. Wallace, and Robert B. Wilson 


\section{AIP | Proceedings}

\section{Estimation of GRB detection by FiberGLAST}

S. Phengchamnan, K. Aisaka, M. Atac, W. R. Binns, J. H. Buckley, M. L. Cherry, D. Cline, P. Dowkontt, J. W. Epstein, M. H. Finger, G. J. Fishman, T. G. Guzik, P. L. Hink, M. H. Israel, S. C. Kappadath, G. R. Karr, R. M. Kippen, J. Macri, R. S. Mallozzi, M. L. McConnell, Y. Pischalnikov, W. S. Paciesas, T. A. Parnell, G. N. Pendleton , R. D. Preece, G. A. Richardson, K. Rielage, J. M. Ryan, J. G. Stacy, T. O. Tümer, D. B. Wallace, and R. B. Wilson

Citation: AIP Conference Proceedings 526, 706 (2000); doi: 10.1063/1.1361626

View online: http://dx.doi.org/10.1063/1.1361626

View Table of Contents: http://scitation.aip.org/content/aip/proceeding/aipcp/526?ver=pdfcov

Published by the AIP Publishing

\section{Articles you may be interested in}

GRB Coordinates Network (GCN): A status report

AIP Conf. Proc. 526, 731 (2000); 10.1063/1.1361631

Progress incorporating the NEAR mission into the interplanetary GRB network AIP Conf. Proc. 526, 726 (2000); 10.1063/1.1361630

MARGIE: A gamma-ray burst ultra-long duration balloon mission

AIP Conf. Proc. 526, 696 (2000); 10.1063/1.1361624

Observations of gamma-ray bursts with MAXI on the international space station

AIP Conf. Proc. 526, 691 (2000); 10.1063/1.1361623

The INTEGRAL burst alert system

AIP Conf. Proc. 526, 686 (2000); 10.1063/1.1361622 


\title{
Estimation of GRB Detection by FiberGLAST
}

S. Phengchamnan ${ }^{1}$, K. Aisaka ${ }^{2}$, M. Atac ${ }^{2}$, W.R. Binns ${ }^{3}$, J.H. Buckley ${ }^{3}$, M.L. Cherry ${ }^{4}$, D. Cline ${ }^{2}$, P. Dowkontt ${ }^{3}$, J.W. Epstein ${ }^{3}$, M.H. Finger ${ }^{5,9}$, G.J. Fishman ${ }^{5}$, T.G. Guzik ${ }^{4}$, P.L. Hink ${ }^{3}$, M.H. Israel ${ }^{3}$, S.C. Kappadath ${ }^{3}$, G.R. Karr ${ }^{1}$, R.M. Kippen ${ }^{1}$, J. Macri ${ }^{6}$, R.S. Mallozzi ${ }^{1}$, M.L. McConnell ${ }^{6}$, Y. Pischalnikov ${ }^{2}$, W.S. Paciesas ${ }^{1}$, T.A. Parnell ${ }^{1}$, G.N. Pendleton ${ }^{1}$, R.D. Preece ${ }^{1}$, G.A. Richardson ${ }^{1}$, K. Rielage ${ }^{3}$, J.M. Ryan ${ }^{6}$, J.G. Stacy ${ }^{2,3}$, T.O. Tümer ${ }^{9}$, D.B. Wallace ${ }^{1}$, and R.B. Wilson ${ }^{5}$

\author{
${ }^{1}$ University of Alabuma in Huntsville, Huntsville, AL 35899, USA \\ ${ }^{2}$ Department of Physics and Astronomy, University of California, \\ Los Angeles, CA 90095, USA \\ ${ }^{3}$ Department of Physics 6 McDonnell Ctr. for Space Sciences, \\ Washington Univ., St. Louis, MO 63130,USA \\ ${ }^{4}$ Department of Physics, Louisiana State Univ., Baton Rouge, LA 70803, USA \\ ${ }^{5}$ NASA/Marshall Space Flight Center, Huntsville, AL 35812, USA \\ ${ }^{6}$ Space Science Center, Univ. of New Hampshire, Durham, NH 03824, USA \\ ${ }^{7}$ Southern Univ., Baton Rouge, LA 70813, USA \\ ${ }^{8}$ Institute of Geophysics \& Planetary Physics, Univ. of California, \\ Riverside, CA 92521, USA \\ ${ }^{9}$ Universities Space Research Association
}

\begin{abstract}
FiberGLAST is one of several instrument concepts being developed for possible inclusion as the primary Gamma-ray Large Area Space Telescope (GLAST) instrument. The predicted FibcrGL $\Lambda$ ST effective area is more than $12,000 \mathrm{~cm}^{2}$ for energies between $30 \mathrm{MeV}$ and $300 \mathrm{GeV}$, with a field of view that is essentially flat from $0^{\circ}-80^{\circ}$. The detector will achieve a sensitivity more than 10 times that of EGRET. We present results of simulations that illustrate the sensitivity of FibcrGL $\Lambda$ ST for the detection of gamma-ray bursts.
\end{abstract}

\section{INTRODUCTION}

Understanding the nature of gamma-ray bursts (GRBs) is among the most important science objectives of NASA's GLAST (Gamma-ray Large Area Space Tele-

CP526, Gamma-Ray Bursts: $5^{\text {th }}$ Huntsville Symposium, edited by R. M. Kippen, et al. (C) 2000 American Institute of Physics 1-56396-947-5/00/\$17.00 
scope) mission. Observations by CGRO/EGRET have shown that the high-energy gamma-ray emission can be a significant fraction of the burst fluence, and that burst spectra commonly extend to GeV energies with no evidence for a high-energy cut-ofl [1]. Even more exciting is the observation by EGRET of high-energy burst emission after lower energy gamma rays were no longer detectable, with time delays of as much as 90 minutes [2,3]. Clearly there is a need for high-energy observations of more bursts and with greater sensitivity, and this has been considered in defining the GLAST scientific requirements.

FiberGLAST is one of several concepts being developed for possible inclusion as the primary GLAST instrument. The predicted response of FiberGLAST has been estimated using Monte Carlo simulations. We use extrapolations of BATSE burst spectra along with a simulated response database to estimate the number of burst photons that will be detected by FiberGLAST. The predicted FiberGLAST effective area is more than $12,000 \mathrm{~cm}^{2}$ for energies between $30 \mathrm{MeV}$ and $100 \mathrm{GeV}$, with a field of view that is essentially flat from $0^{\circ}-80^{\circ}$. The FiberGLAST instrument concept is described in more detail by Rielage et al. [4].

\section{FIRST METHOD}

This method is a rough estimation of the number of GRBs that will be detected by FiberGLAST. The energy range that FiberGLAST will observe is from $10 \mathrm{MeV}$ to $300 \mathrm{GeV}$, and the effective area is $\sim 12,000 \mathrm{~cm}^{2}$ up to an $80^{\circ}$ viewing angle. We also assume Band's GRB spectral model with $\alpha=-1, \beta=-2$ and $E_{0}=200 \mathrm{keV}$. Since the effective area of FiberGLAST depends on energy, we divide our peak flux calculation into 10 different encrgy bins. Using 5 counts $\cdot \mathrm{s}^{-1}$ as the minimum detection critcria and by comparing our calculation value with the $\log N-\log P$ of GRBs that BATSE has detected [5], we can estimate how many GRBs we would expect to detect. $\log N-\log P$ on the $1024 \mathrm{~ms}$ time scale is used to extrapolate the number of GRBs. Our calculation yields the result of 549 bursts per years that FiberGLAST might detect.

We checked the validity of this simulation by repeating the same procedure using the EGRET response [8]. During its first 3.4 years, EGRET detected five bursts, each with at least seven spark chamber events above $30 \mathrm{MeV}$ (during the gammaray active phase as defined by BATSE). Assuming $30 \%$ observing efficiency for EGRET, this methodology predicts twenty-four detected bursts in 3.4 years, but EGRET observed only 5 GRBs. We can see that this simple method overestimates the number of GRBs that might be detected by FiberGLAST. We will show another method in the next section that yields a more reliable estimate.

\section{SECOND METHOD}

In this method, in order to simulate the response of FibcrGLAST to GRBs, we use the measured distribution of BATSE burst spectra, and extrapolate them to 
higher energies. The high-energy gamma-ray flux from a GRB of a given BATSE peak flux or fluence was estimated using the catalog of Preece et al. $[6,7]$, which includes time-resolved spectral fits for a large sample of bright BATSE bursts. We chose for our spectral templates 102 events from this catalog that also had BATSE peak flux and fluence measurements. For most burst sub-intervals in the catalog, the Band GRB function, or other broken broken power-law models, provided an acceptable fit. For each of these, we extrapolated the best-fit high-energy powerlaw spectral model to the FiberGLAST energy range and computed a fluence for each burst by summing over all such sub-intervals. For a few sub-intervals no high-energy power-law could be determined, so the FiberGLAST fluence was set to zero. Fluences were computed in five energy bins between $10 \mathrm{MeV}$ and $300 \mathrm{GeV}$. We consider this method to be more robust than extrapolating a single spectrum averaged over each burst. However, due to the finite BATSE energy bandwidth, there is some concern that the high-energy power law spectral index determined from BATSE spectra is systematically too hard. Some evidence for this comes from the comparison of the distribution of BATSF high-energy spectral indices with COMPTEL observations of GRB spectra in the $0.75-30 \mathrm{MeV}$ range. The mean COMPTEL spectral index is -2.53 [9], whereas the BATSE high-energy spectral index distribution peaks around -2.25 , though with a pronounced skewness toward softer spectra. For better consistency, we arbitrarily softened each BATSE spectrum by adding -0.2 to the spectral index before extrapolation.

We generated two sets of simulated bursts by first picking a peak flux $P_{B}$ (1.024 s timescale) or fluence $S_{B}(25-2000 \mathrm{keV})$ randomly from the observed BATSE $\log N-\log P_{B}$ and $\log N-\log S_{B}$ distributions and then computing the FiberGLAST fluence $S_{F}$ that each template burst would have if it were scaled to the chosen flux or fluence:

$$
S_{F}=\frac{P_{B}}{P_{B}^{\iota}} S_{F}^{t} \quad \text { and } \quad S_{F}=\frac{S_{B}}{S_{B}^{\iota}} S_{F}^{t},
$$

respectively, where $P_{B}^{t}$ and $S_{B}^{t}$ are the BATSE peak flux and fluence of a template burst, and $S_{F}^{t}$ is its extrapolated FiberGLAST fluence. A random direction of incidence (within $90^{\circ}$ of the FiberGLAST primary axis) was chosen for each such event, and the extrapolated fluence $S_{F}$ was convolved with the simulated FiberGLAST response to determine the total detected counts in each of five energy bins: 10$30 \mathrm{MeV}, 30-100 \mathrm{MeV}, 0.1-1 \mathrm{GeV}, 1-10 \mathrm{GeV}$, and 10-300 GeV. For each simulated set, 1,292 BATSE values were used, so the total number of simulated bursts in each set is $1.292 \times 102=131,784$.

We checked the validity of these simulations by repeating the same procedure using the EGRET response (as in the first method). This second methodology predicts nine EGRET detections if we use peak flux scaling, and six if we use fluence scaling, whereas five bursts were actually detected. Given the small numbers, both techniques produce reasonably consistent results - giving us confidence in the FiberGLAST predictions using this method. 
TABLE 1. Burst detectability using peak flux scaling

\begin{tabular}{lcccccc}
\multirow{2}{*}{$\begin{array}{l}\text { Energy } \\
(\mathrm{GeV})\end{array}$} & \multicolumn{6}{c}{ Fraction of unocculted bursts (percent) } \\
\cline { 2 - 7 } & $\begin{array}{c}<3 \\
\text { photons }\end{array}$ & $\begin{array}{c}3-10 \\
\text { photons }\end{array}$ & $\begin{array}{c}10-10^{2} \\
\text { photons }\end{array}$ & $\begin{array}{c}10^{2}-10^{3} \\
\text { photons }\end{array}$ & $\begin{array}{c}10^{3}-10^{4} \\
\text { photons }\end{array}$ & $\begin{array}{c}>10^{4} \\
\text { photons }\end{array}$ \\
\hline $0.01-0.03$ & 37 & 12 & 32 & 16 & 2.5 & 0.1 \\
$0.03-0.1$ & 39 & 12 & 31 & 15 & 2.6 & 0.1 \\
$0.1-1$ & 48 & 15 & 25 & 10 & 1.6 & 0.1 \\
$1-10$ & 77 & 10 & 10 & 2.5 & 0.2 & 0 \\
$10-300$ & 93 & 4.0 & 2.4 & 0.3 & 0 & 0 \\
\hline $0.01-300$ & 34 & 7.5 & 28 & 23 & 6.4 & 0.6 \\
\hline \hline
\end{tabular}

TABLE 2. Burst occurrence rate using peak flux scaling

\begin{tabular}{|c|c|c|c|c|c|}
\hline \multirow{2}{*}{$\begin{array}{l}\text { Energy } \\
(\mathrm{GeV})\end{array}$} & \multicolumn{5}{|c|}{ Rate (bursts per year) } \\
\hline & $\begin{array}{c}3-10 \\
\text { photons }\end{array}$ & $\begin{array}{l}10-10^{2} \\
\text { photons }\end{array}$ & $\begin{array}{l}10^{2}-10^{3} \\
\text { photons }\end{array}$ & $\begin{array}{l}10^{3}-10^{4} \\
\text { photons }\end{array}$ & $\begin{array}{l}>10^{4} \\
\text { photons }\end{array}$ \\
\hline$\overline{0.01-0.03}$ & 52 & 143 & 73 & 11 & 0.4 \\
\hline $0.03-0.1$ & 54 & 136 & 69 & 12 & 0.6 \\
\hline $0.1-1$ & 68 & 110 & 46 & 7.2 & 0.3 \\
\hline $1-10$ & 46 & 45 & 11 & 1.1 & 0 \\
\hline $10-300$ & 18 & 11 & 1.4 & 0 & 0 \\
\hline$\overline{0.01-300}$ & 33 & 126 & 103 & 29 & 2.6 \\
\hline
\end{tabular}

TABLE 3. Burst detectability using fluence scaling

\begin{tabular}{lcccccc}
\hline \multirow{2}{*}{$\begin{array}{l}\text { Energy } \\
(\mathrm{GeV})\end{array}$} & \multicolumn{6}{c}{ Fraction of unocculted bursts } \\
\cline { 2 - 7 } & $\begin{array}{c}<3 \\
\text { photons }\end{array}$ & $\begin{array}{c}3-10 \\
\text { photons }\end{array}$ & $\begin{array}{c}10-10^{2} \\
\text { photons }\end{array}$ & $\begin{array}{c}10^{2}-10^{3} \\
\text { photons }\end{array}$ & $\begin{array}{c}10^{3}-10^{4} \\
\text { photons }\end{array}$ & $\begin{array}{c}>10^{4} \\
\text { photons }\end{array}$ \\
\hline $0.01-0.03$ & 46 & 14 & 26 & 12 & 1.6 & 0 \\
$0.03-0.1$ & 48 & 14 & 25 & 11 & 1.6 & 0 \\
$0.1-1$ & 58 & 14 & 20 & 7.1 & 0.9 & 0 \\
$1-10$ & 83 & 8.5 & 7.4 & 1.5 & 0.1 & 0 \\
$10-300$ & 96 & 2.7 & 1.5 & 0.1 & 0 & 0 \\
\hline $0.01-300$ & 41 & 11 & 26 & 17 & 4.3 & 0.3 \\
\hline \hline
\end{tabular}

TABLE 4. Burst occurrence rate using fluence scaling

\begin{tabular}{|c|c|c|c|c|c|}
\hline \multirow{2}{*}{$\begin{array}{l}\text { Energy } \\
(\mathrm{GeV})\end{array}$} & \multicolumn{5}{|c|}{ Rate (bursts per year) } \\
\hline & $\begin{array}{c}3-10 \\
\text { photons }\end{array}$ & $\begin{array}{l}10-10^{2} \\
\text { photons }\end{array}$ & $\begin{array}{l}10^{2}-10^{3} \\
\text { photons }\end{array}$ & $\begin{array}{l}10^{3}-10^{4} \\
\text { photons }\end{array}$ & $\begin{array}{l}>10^{4} \\
\text { photons }\end{array}$ \\
\hline$\overline{0.01-0.03}$ & 64 & 115 & 52 & 7.1 & 0 \\
\hline $0.03-0.1$ & 64 & 109 & 49 & 7.2 & 0 \\
\hline $0.1-1$ & 63 & 87 & 32 & 4.1 & 0 \\
\hline $1-10$ & 38 & 33 & 6.7 & 0.4 & 0 \\
\hline $10-300$ & 12 & 6.7 & 0.6 & 0 & 0 \\
\hline $0.01-300$ & 50 & 117 & 77 & 19 & 1.3 \\
\hline
\end{tabular}




\section{DISCUSSION}

Our first method is a rough estimation that most likely overestimates the number of GRBs that FiberGLAST should detect. The second method gives us better estimation. From Tables 1-4, it can be seen that FiberGLAST will detect at least 10 photons each from roughly half of the unocculted bursts above BATSE's threshold, and roughly one in five bursts should produce at least 100 photons in FiberGLAST. Bursts that produce at least 10 photons above $10 \mathrm{GeV}$ should be detected once every month or two, and there is a good chance of detecting at least 100 photons above $10 \mathrm{GeV}$ from a burst during the GLAST mission. The photons above $10 \mathrm{GeV}$ are important not only for their direct physical implications, but also because their directions can be more accurately determined.

These simulations assume no spcctral cut-offs and do not consider additional photons from the delayed/extended emission observed by EGRET. The large predicted numbers of bursts with more than 10 photons per energy bin should allow measurement of spectral breaks or cut offs up to at least $10 \mathrm{GeV}$ if they exist, even for the "wide-field" bursts for which FiberGLAST has worse energy resolution.

\section{REFERENCES}

1. Dingus, B. L., Catelli, J. R., \& Schneid, E. J., in Gamma-Ray Bursts: 4th Huntsville Symposium, AIP CP428, Ed. C. A. Meegan et al., New York: AIP, 1998, pp. 349.

2. Dingus, B. L., in Gamma-Ray Bursts: 2nd Huntsville Symposium, AIP CP307. Ed. G. J. Fishman et al., New York: AIP, 1994, pp. 22.

3. Hurley, K., Nature 372, 652 (1994).

4. Rielage, K., in Proc. 26th Intl. Cosmic ray Conf., Salt Lake City, paper OG4.2.21, in press (1999).

5. Pendleton, G. N., Ap.J 464, 606 (1996).

6. Preece, R. D., 1998, ApJ 496, 849 (1998).

7. Preece, R. D., ApJS 496, submitted (1999).

8. Thompson, D. J., ApJS 86, 629 (1998).

9. Kippen, R. M., Adv. Sp. Res. 22, 1097 (1998). 